\title{
Dynamics of a Multigroup SIR Epidemic Model with Nonlinear Incidence and Stochastic Perturbation
}

\author{
Yuguo Lin ${ }^{1,2}$ and Daqing Jiang ${ }^{1}$ \\ ${ }^{1}$ School of Mathematics and Statistics, Northeast Normal University, Changchun, Jilin 130024, China \\ ${ }^{2}$ School of Mathematics, Beihua University, Jilin, Jilin 132013, China
}

Correspondence should be addressed to Daqing Jiang; daqingjiang2010@hotmail.com

Received 18 April 2013; Accepted 20 June 2013

Academic Editor: Andrei Korobeinikov

Copyright (C) 2013 Y. Lin and D. Jiang. This is an open access article distributed under the Creative Commons Attribution License, which permits unrestricted use, distribution, and reproduction in any medium, provided the original work is properly cited.

We introduce stochasticity into a multigroup SIR model with nonlinear incidence. We prove that when the intensity of white noise is small, the solution of stochastic system converges weakly to a singular measure (i.e., a distribution) if $\mathscr{R}_{0} \leq 1$ and there exists an invariant distribution which is ergodic if $\mathscr{R}_{0}>1$. This is the same situation as the corresponding deterministic case. When the intensity of white noise is large, white noise controls this system. This means that the disease will extinct exponentially regardless of the magnitude of $\mathscr{R}_{0}$.

\section{Introduction}

Considering different contact patterns, distinct number of sexual partners, or different geography, and so forth, individual hosts are often divided into groups in modeling epidemic diseases. Multigroup models have been proposed in the literature to describe the transmission dynamics of infectious diseases in heterogeneous host populations. One of the earliest work on multigroup models is the seminal paper by Lajmanovich and Yorke [1] on a class of SIS multigroup models for the transmission dynamics of Gonorrhea. From then on, much research has been done on various forms of multigroup models, see, for example, [2-6]. It is well known that the global stability of the endemic equilibrium of multigroup models is a very challenging problem. Recently, Guo et al. [7, 8] and Li and Shuai [9] proposed a graph-theoretic approach to the method of global Lyapunov functions and completely solved this problem for some multigroup epidemic models. Subsequently, this approach or ideas of [7-9] were applied to the investigation into the dynamics of several classes of multigroup epidemic models (e.g., [10-13]).

Now we consider a deterministic multigroup SIR (susceptible, infective, and recovered) epidemic model with nonlinear incidence:

$$
\dot{S}_{k}=\Lambda_{k}-\sum_{j=1}^{n} \beta_{k j} S_{k} \phi_{j}\left(I_{j}\right)-d_{k} S_{k}
$$

$$
\begin{aligned}
\dot{I}_{k} & =\sum_{j=1}^{n} \beta_{k j} S_{k} \phi_{j}\left(I_{j}\right)-\left(\epsilon_{k}+\gamma_{k}\right) I_{k}, \\
\dot{R}_{k} & =\gamma_{k} I_{k}-\delta_{k} R_{k}, \quad k=1,2, \ldots, n .
\end{aligned}
$$

Here $S_{k}(t), I_{k}(t)$, and $R_{k}(t)$ denote the susceptible, infective, and recovered population at time $t$ in the $k$ th group, respectively, $k=1,2, \ldots, n$. Suppose the death rates of $S_{k}, I_{k}, R_{k}$ in the $k$ th group are different. The parameters in the model (1) are assumed to be positive and summarized in the following list:

$\Lambda_{k}$ : influx of individuals into the $k$ th group;

$\beta_{k j}$ : transmission coefficient between compartments $S_{k}$ and $I_{j}$;

$d_{k}$ : death rate of $S$ compartment in the $k$ th group;

$\epsilon_{k}$ : death rate of $I$ compartment in the $k$ th group;

$\delta_{k}$ : death rate of $R$ compartment in the $k$ th group;

$\gamma_{k}$ : recovery rate of infectious individuals in the $k$ th group.

Considering that the death rates of infective and recovered population are usually no less than the susceptible's, we assume $d_{k} \leq \min \left\{\epsilon_{k}, \delta_{k}\right\}$ for all $k$. 
Throughout this paper, we consider the following basic assumptions on functions $\phi_{j}(x), x \in(0,+\infty), j=1,2, \ldots, n$ :

$\left(C_{1}\right) \phi_{j}(x)$ is a $C^{1}$-function on $(0,+\infty), \phi_{j}(x)>0$ for $x>0$
$\quad$ and $\phi_{j}(0)=0 ;$

$\left(C_{2}\right)$ there exist positive constants $c_{j}$ such that $\phi_{j}(x) \leq c_{j} x$, $x \in(0,+\infty)$;

$\left(C_{3}\right) \lim _{x \rightarrow 0^{+}}\left(\phi_{j}(x) / x\right)=c_{j}$

$\left(C_{4}\right)\left[\phi_{j}(x)-\phi_{j}(y)\right]\left[\phi_{j}(x) / x-\phi_{j}(y) / y\right] \leq 0$, for any $x>0$ and $y>0$.

It is easy to check that classes of $\phi_{j}(x)$ satisfying $\left(C_{1}\right)-\left(C_{3}\right)$ include common incidence functions such as $\phi_{j}\left(I_{j}\right)=I_{j}$ [7], $\phi_{j}\left(I_{j}\right)=I_{j} /\left(1+\alpha_{j} I_{j}\right)[14], \phi_{j}\left(I_{j}\right)=I_{j} /\left(1+\alpha I_{j}^{2}\right)[15]$. Furthermore, $\phi_{j}\left(I_{j}\right)=I_{j}, \phi_{j}\left(I_{j}\right)=I_{j} /\left(1+\alpha_{j} I_{j}\right)$ satisfy $\left(C_{4}\right)$.

In Sun [13], the author considered a general multigroup SIR models with nonlinear incidence by using the same method as in [9]. Noting that model (1) is a special case of multigroup SIR models appeared in [13], according to the results of [13], the following results hold for system (1).

Proposition 1. Assume that $B=\left(\beta_{k j}\right)$ is irreducible and $\phi_{j}(x)$ satisfies $\left(C_{1}\right)-\left(C_{3}\right)$.

(1) If $\mathscr{R}_{0} \leq 1$, then for system (1), $P_{0}=\left(S_{1}^{0}, 0,0, S_{2}^{0}\right.$, $\left.0,0, \ldots, S_{n}^{0}, 0,0\right)$ is the unique equilibrium and it globally asymptotically stable in $\Gamma$;

(2) if $\mathscr{R}_{0}>1$ and $\left(C_{4}\right)$ is satisfied, then there exists an endemic equilibrium $P^{*}=\left(S_{1}^{*}, I_{1}^{*}, R_{1}^{*}, \ldots, S_{n}^{*}, I_{n}^{*}, R_{n}^{*}\right)$ for system (1) and $P^{*}$ is globally asymptotically stable in $\Gamma$,

where $S_{k}^{0}=\Lambda_{k} / d_{k}, k=1,2, \ldots, n, \mathscr{R}_{0}=\rho\left(M_{0}\right)$ (the spectral radius of $\left.M_{0}\right), M_{0}=M\left(S_{1}^{0}, S_{2}^{0}, \ldots, S_{n}^{0}\right)=\left(\beta_{k j} S_{k}^{0} c_{j} /\right.$, $\left.\left(\epsilon_{k}+\gamma_{k}\right)\right)_{n \times n}$, and $\Gamma=\left\{\left(S_{1}, I_{1}, R_{1}, \ldots, S_{n}, I_{n}, R_{n}\right) \in \mathbb{R}_{+}^{3 n}:\right.$ $\left.S_{k} \leq \Lambda_{k} / d_{k}, S_{k}+I_{k} \leq \Lambda_{k} / d_{k}, k=1,2, \ldots, n\right\}$.

The aim of this paper is to evaluate the effect of stochastic parameter perturbation on system (1). Considering the existence of environmental noise, we introduce randomness into the model (1) by replacing the parameters $d_{k}, \epsilon_{k}$, and $\delta_{k}$ by

$$
\begin{aligned}
& d_{k} \longrightarrow d_{k}+\alpha_{k} \dot{B}_{1 k}(t), \\
& \epsilon_{k} \longrightarrow \epsilon_{k}+\beta_{k} \dot{B}_{2 k}(t), \\
& \delta_{k} \longrightarrow \delta_{k}+\sigma_{k} \dot{B}_{3 k}(t),
\end{aligned}
$$

where $B_{1 k}(t), B_{2 k}(t), B_{3 k}(t), k=1,2, \ldots, n$ are mutual independent standard Brownian motions with $B_{1 k}(0)=$ $0, B_{2 k}(0)=0, B_{3 k}(0)=0$, and the intensities of white noises $\alpha_{k}^{2}>0, \beta_{k}^{2}>0, \sigma_{k}^{2}>0$, respectively. Then the stochastic version corresponding to the deterministic model (1) takes the following form:

$$
\begin{aligned}
& d S_{k}(t)= {\left[\Lambda_{k}-\sum_{j=1}^{n} \beta_{k j} S_{k}(t) \phi_{j}\left(I_{j}(t)\right)-d_{k} S_{k}(t)\right] d t } \\
&-\alpha_{k} S_{k}(t) d B_{1 k}(t), \\
& d I_{k}(t)= {\left[\sum_{j=1}^{n} \beta_{k j} S_{k}(t) \phi_{j}\left(I_{j}(t)\right)-\left(\epsilon_{k}+\gamma_{k}\right) I_{k}(t)\right] d t } \\
&-\beta_{k} I_{k}(t) d B_{2 k}(t), \\
& d R_{k}(t)= {\left[\gamma_{k} I_{k}(t)-\delta_{k} R_{k}(t)\right] d t-\sigma_{k} R_{k}(t) d B_{3 k}(t), } \\
& k=1,2, \ldots, n .
\end{aligned}
$$

System (3) with bilinear incidence has been researched by Ji et al. [16]. They proved that if $\mathscr{R}_{0} \leq 1$, the solution of the model is fluctuating around a steady state, which is the disease-free equilibrium of the corresponding deterministic model; while if $\mathscr{R}_{0}>1$, there is a stationary distribution, which means the disease will prevail.

In this paper, we will prove that when the intensity of white noise is small, the solution of system (3) converges weakly to a singular measure (i.e., a distribution) if $\mathscr{R}_{0} \leq 1$ and there exists an invariant distribution which is ergodic if $\mathscr{R}_{0}>1$. This is the same situation as in Proposition 1 . When the intensity of white noise is large, the disease will extinct exponentially regardless of the magnitude of $\mathscr{R}_{0}$. Compared to the results of Ji et al. [16] our results can provide a deep insight into the dynamics of corresponding multigroup model.

It is worth mentioning that by now there are many excellent works about stochastic single-group SIR model. Beretta et al. [17] considered a stochastic SIR model with time delays and obtained asymptotic mean square stability conditions for positive equilibrium. In this paper, the authors assumed that stochastic perturbations are of white noise type, which are directly proportional to distances $S(t), I(t), R(t)$ from values of $S^{*}, I^{*}, R^{*}$, influence on the $\dot{S}(t), \dot{I}(t), \dot{R}(t)$, respectively. Tornatore et al. [18] discuss a single-group case of (3). They proved that $0<\beta<\min \left\{\lambda+\mu-\sigma^{2} / 2,2 \mu\right\}$ is a sufficient condition for the asymptotic stability of the disease-free equilibrium. And only by computer simulations they showed that if $\min \left\{\lambda+\mu-\sigma^{2} / 2,2 \mu\right\}<\beta<\lambda+\mu+$ $\sigma^{2} / 2$, the disease-free equilibrium is stable and the disease does not occur; if $(\lambda+\mu)\left(R_{0}-1\right)>\sigma^{2} / 2$, the diseasefree equilibrium is unstable. Ji et al. [19] considered the same model as in [18]. They deduce the globally asymptotical stability and exponential mean square stability of the diseasefree equilibrium under some conditions and investigate the asymptotic behavior of the solution around the endemic equilibrium of the deterministic model.

The rest of this paper is organized as follows. In Section 2, we show there is a unique nonnegative solution of system (3). In Section 3 , if $\mathscr{R}_{0} \leq 1$ combined with small or large enough intensity of white noise, we show that the solution converges 
weakly to a singular measure. Section 4 focuses on the persistence of the disease. By choosing appropriate Lyapunov function, we show that there is a stationary distribution for system (3) and it is ergodic, if $\mathscr{R}_{0}>1$. Finally, for the self-contained, we present an Appendix which contains some results used in the previous sections.

Throughout this paper, let $\left(\Omega, \mathscr{F},\left\{\mathscr{F}_{t}\right\}_{t \geq 0}, P\right)$ be a complete probability space with a filtration $\left\{\mathscr{F}_{t}\right\}_{t \geq 0}$ satisfying the usual conditions (i.e., it is right continuous and $\mathscr{F}_{0}$ contains all $P$-null sets). Denote

$$
\begin{aligned}
& \mathbb{R}_{+}^{3 n}=\left\{x \in \mathbb{R}^{3 n}: x_{k}>0 \forall 1 \leq k \leq 3 n\right\}, \\
& \overline{\mathbb{R}}_{+}^{3 n}=\left\{x \in \mathbb{R}^{3 n}: x_{k} \geq 0 \quad \forall 1 \leq k \leq 3 n\right\} .
\end{aligned}
$$

In general, consider a $d$-dimensional stochastic differential equation

$$
d x(t)=f(x(t), t) d t+g(x(t), t) d B(t) \quad \text { on } t \geq t_{0}
$$

with initial value $x\left(t_{0}\right)=x_{0} \in \mathbb{R}^{d} . B(t)$ denotes $d$ dimensional standard Brownian motion defined on the above probability space. Define the differential operator $L$ associated with (5) by

$$
\begin{aligned}
L= & \frac{\partial}{\partial t}+\sum_{k=1}^{d} f_{k}(x, t) \frac{\partial}{\partial x_{k}} \\
& +\frac{1}{2} \sum_{k, j=1}^{d}\left[g^{T}(x, t) g(x, t)\right]_{k j} \frac{\partial^{2}}{\partial x_{k} \partial x_{j}} .
\end{aligned}
$$

If $L$ acts on a function $V \in C^{2,1}\left(R^{d} \times \overline{\mathbb{R}}_{+} ; \overline{\mathbb{R}}_{+}\right)$, then

$$
\begin{aligned}
L V(x, t)= & V_{t}(x, t)+V_{x}(x, t) f(x, t) \\
& +\frac{1}{2} \operatorname{trace}\left[g^{T}(x, t) V_{x x}(x, t) g(x, t)\right],
\end{aligned}
$$

where $V_{t}=\partial V / \partial t, V_{x}=\left(\partial V / \partial x_{1}, \ldots, \partial V / \partial x_{d}\right), V_{x x}=$ $\left(\partial^{2} V / \partial x_{k} \partial x_{j}\right)_{d \times d}$. By Itô's formula, if $x(t) \in R^{d}$, then

$$
\begin{aligned}
d V(x(t), t)= & L V(x(t), t) d t \\
& +V_{x}(x(t), t) g(x(t), t) d B(t) .
\end{aligned}
$$

Consider (5), assume $f(0, t)=0$ and $g(0, t)=0$ for all $t \geq$ $t_{0}$. So $x(t) \equiv 0$ is a solution of (5), called the trivial solution or equilibrium position.

\section{Existence and Uniqueness of the Nonnegative Solution}

For a population model, one is interested in whether the solution is nonnegative and global. Hence in this section we show that the solution of system (3) is global and nonnegative. By making the change of variables and Lyapunov analysis method (see [20]), we will show global existence and uniqueness of the positive solution. From now on, we denote the solution $\left(S_{1}(t), I_{1}(t), R_{1}(t), \ldots, S_{n}(t), I_{n}(t), R_{n}(t)\right)$ of system (3) as $Y(t)$.
Theorem 2. If assumption $\left(C_{1}\right)$ holds and $B=\left(\beta_{k j}\right)_{n \times n}$ is irreducible, then for any initial value $Y(0) \in R_{+}^{3 n}$, there is a unique solution $Y(t)$ of system (3) on $t \geq 0$, and the solution will remain in $R_{+}^{3 n}$ with probability 1 .

Proof. First consider system

$$
\begin{aligned}
& d u_{k}(t)= {\left[\Lambda_{k} e^{-u_{k}(t)}-\sum_{j=1}^{n} \beta_{k j} \phi_{j}\left(e^{v_{j}(t)}\right)-d_{k}-\frac{\alpha_{k}^{2}}{2}\right] d t } \\
&-\alpha_{k} d B_{1 k}(t), \\
& d v_{k}(t)=[ {\left[\sum_{j=1}^{n} \beta_{k j} e^{u_{k}(t)-v_{k}(t)} \phi_{j}\left(e^{v_{j}(t)}\right)-\left(\epsilon_{k}+\gamma_{k}\right)-\frac{\beta_{k}^{2}}{2}\right] d t } \\
&-\beta_{k} d B_{2 k}(t), \\
& d w_{k}(t)= {\left[\gamma_{k} e^{v_{k}(t)}-\delta_{k} w_{k}(t)\right] d t-\sigma_{k} w_{k}(t) d B_{3 k}(t), } \\
& k=1,2, \ldots, n .
\end{aligned}
$$

With initial value $u_{k}(0)=\ln S_{k}(0), v_{k}(0)=\ln I_{k}(0)$, $w_{k}(0)=R_{k}(0), 1 \leq k \leq n$. Noting that $\left(C_{1}\right)$ holds, the coefficients of system (9) satisfy the local Lipschitz condition; then there is a unique local solution $\left(u_{1}(t), v_{1}(t), w_{1}(t), \ldots, u_{n}(t), v_{n}(t), w_{n}(t)\right)$ on $t \in\left[0, \tau_{e}\right)$, where $\tau_{e}$ is the explosion time. Therefore, by Itô's formula, it is easy to see $\left(e^{u_{1}(t)}, e^{v_{1}(t)}, w_{1}(t), \ldots, e^{u_{n}(t)}, e^{v_{n}(t)}, w_{n}(t)\right)$ is the unique positive local solution to system (3) with initial value $Y(0) \in R_{+}^{3 n}$.

Next, we will prove that this solution is global. The following proof is almost the same as in the proof of Theorem 3.1 in [16]. We do not alter any words except replacing $I_{j}$ by $\phi_{j}\left(I_{j}\right)$ and therefore we can obtain that $L V \leq \widetilde{M}$. Here we omit the details.

\section{Exponential Stability of Infectious Disease}

It is clear that $P_{0}=\left(\Lambda_{1} / d_{1}, 0,0, \Lambda_{2} / d_{2}, 0,0, \ldots, \Lambda_{n} / d_{n}, 0,0\right)$ is the disease-free equilibrium of system (1) but not (3). For system (1), which has been mentioned in Introduction, $P_{0}$ is globally stable if $\mathscr{R}_{0} \leq 1$, which means the disease will die out after some period of time. Hence, it is interesting to study the disease-free equilibrium for controlling infectious disease. Although there is none of disease-free equilibrium of system (3), in this section, we can still present sufficient conditions for the disease to extinct exponentially.

Theorem 3. Assume $\left(C_{1}\right),\left(C_{2}\right)$ hold and $B=\left(\beta_{k j}\right)_{n \times n}$ is irreducible. If $2 d_{k}>\alpha_{k}^{2}, k=1,2, \ldots, n$, then the solution $Y(t)$ of system (3) with initial value $Y(0) \in R_{+}^{3 n}$ has the property 
$\lim _{t \rightarrow+\infty}\left(\log \left(\sum_{k=1}^{n} a_{k} I_{k}\right) / t\right) \leq c$, a.s. If $c<0$, the disease will extinct exponentially almost surely. Here

$$
\begin{gathered}
M_{0}=M\left(S^{0}\right)=\left(\frac{\beta_{k j} S_{k}^{0} c_{j}}{\epsilon_{k}+\gamma_{k}}\right)_{n \times n}, \quad S_{k}^{0}=\frac{\Lambda_{k}}{d_{k}}, \\
a_{k}=\frac{\omega_{k}}{\left(\epsilon_{k}+\gamma_{k}\right)}, \quad k=1,2, \ldots, n, \\
\left(\omega_{1}, \omega_{2}, \ldots, \omega_{n}\right) \text { is a left eigenvector } \\
\quad \text { of } M_{0} \text { corresponding to } \rho\left(M_{0}\right), \\
+\max _{0 \leq k, j \leq n}\left\{\frac{\beta_{k j} c_{j}}{a_{j}}\right\} \sum_{k=1}^{n} \frac{a_{k} \alpha_{k} S_{k}^{0}}{\sqrt{2 d_{k}-\alpha_{k}^{2}}} \\
+\min _{1 \leq k \leq n}\left\{\epsilon_{k}+\gamma_{k}\right\}\left(\mathscr{R}_{0}-1\right) \mathbf{1}_{\mathscr{R}_{0} \leq 1} \\
+\max _{1 \leq k \leq n}\left\{\epsilon_{k}+\gamma_{k}\right\}\left(\mathscr{R}_{0}-1\right) \mathbf{1}_{\mathscr{R}_{0} \geq 1}-\frac{1}{2 \sum_{k=1}^{n}\left(1 / \beta_{k}^{2}\right)} .
\end{gathered}
$$

Proof. Let $X_{k}, 1 \leq k \leq n$ be the solution of the equation:

$$
\begin{aligned}
d X_{k}(t) & =\left[\Lambda_{k}-d_{k} X_{k}(t)\right] d t-\alpha_{k} X_{k}(t) d B_{1 k}(t), \\
X_{k}(0) & =S_{k}(0) .
\end{aligned}
$$

By comparison theorem for stochastic equations, we have

$$
S_{k}(t) \leq X_{k}(t), \quad \text { a.s. }
$$

It is easy to know that $X_{k}(t)$ has the following property:

$$
\begin{gathered}
\lim _{t \rightarrow \infty} \frac{\int_{0}^{t} X_{k} d t}{t}=S_{k}^{0} \quad \text { a.s., } \\
\lim _{t \rightarrow \infty} \frac{\int_{0}^{t}\left|X_{k}-S_{k}^{0}\right| d t}{t} \leq \frac{\alpha_{k} S_{k}^{0}}{\sqrt{2 d_{k}-\alpha_{k}^{2}}} \\
\text { if } 2 d_{k}>\alpha_{k}^{2}, k=1,2, \ldots, n .
\end{gathered}
$$

As for the proof of the above two properties, the reader may refer to the proof of Theorems 3.1 and 4.1 in [21] for details.

Since $B=\left(\beta_{k j}\right)_{n \times n}$ is irreducible, $\beta_{k j} \geq 0, k, j=1,2, \ldots, n$ and $S_{k}^{0}>0, \epsilon_{k}+\gamma_{k}>0, c_{k}>0, k=1,2, \ldots, n$, then $M_{0}$ is also nonnegative and irreducible. Hence by Lemma A.1, there is a left eigenvector of $M_{0}$ corresponding to $\rho\left(M_{0}\right)$, which is denoted as $\left(\omega_{1}, \omega_{2}, \ldots, \omega_{n}\right)$ and $\omega_{k}>0, k=1,2, \ldots, n$; that is,

$$
\left(\omega_{1}, \omega_{2}, \ldots, \omega_{n}\right) \rho\left(M_{0}\right)=\left(\omega_{1}, \omega_{2}, \ldots, \omega_{n}\right) M_{0}
$$

Let $a_{k}=\omega_{k} /\left(\epsilon_{k}+\gamma_{k}\right), k=1,2, \ldots, n$. Define $C^{2}$-function $V: R_{+}^{n} \rightarrow R_{+}$as follows:

$$
V\left(I_{1}, I_{2}, \ldots, I_{n}\right)=\sum_{k=1}^{n} a_{k} I_{k}
$$

By Itô's formula, we compute

$$
d(\log V)=L(\log V) d t-\frac{1}{V} \sum_{k=1}^{n} a_{k} \beta_{k} I_{k} d B_{2 k}
$$

where

$$
\begin{aligned}
L(\log V)= & \frac{1}{V} \sum_{k=1}^{n} a_{k}\left[\sum_{j=1}^{n} \beta_{k j} S_{k} \phi_{j}\left(I_{j}\right)-\left(\epsilon_{k}+\gamma_{k}\right) I_{k}\right] \\
& -\frac{\sum_{k=1}^{n} a_{k}^{2} \beta_{k}^{2} I_{k}^{2}}{2 V^{2}} \\
\leq & \frac{1}{V} \sum_{k=1}^{n} a_{k}\left[\sum_{j=1}^{n} \beta_{k j} X_{k} c_{j} I_{j}-\left(\epsilon_{k}+\gamma_{k}\right) I_{k}\right] \\
& -\frac{\sum_{k=1}^{n} a_{k}^{2} \beta_{k}^{2} I_{k}^{2}}{2 V^{2}} \\
= & \frac{1}{V} \sum_{k=1}^{n} a_{k}\left[\sum_{j=1}^{n} \beta_{k j} c_{j}\left(X_{k}-S_{k}^{0}\right) I_{j}\right. \\
& -\frac{\sum_{k=1}^{n} a_{k}^{2} \beta_{k}^{2} I_{k}^{2}}{2 V^{2}}
\end{aligned}
$$

according to $\left(C_{2}\right)$ and (12). Let

$$
\begin{gathered}
H_{1}=\frac{1}{V} \sum_{k=1}^{n} a_{k} \sum_{j=1}^{n} \beta_{k j} c_{j}\left(X_{k}-S_{k}^{0}\right) I_{j}, \\
H_{2}=\frac{1}{V} \sum_{k=1}^{n} a_{k}\left[\sum_{j=1}^{n} \beta_{k j} c_{j} S_{k}^{0} I_{j}-\left(\epsilon_{k}+\gamma_{k}\right) I_{k}\right], \\
H_{3}=-\frac{\sum_{k=1}^{n} a_{k}^{2} \beta_{k}^{2} I_{k}^{2}}{2 V^{2}} .
\end{gathered}
$$

Then

$$
\begin{aligned}
H_{1} & \leq \frac{1}{V} \sum_{k=1}^{n} a_{k} \sum_{j=1}^{n} \beta_{k j} c_{j}\left|X_{k}-S_{k}^{0}\right| I_{j} \\
& =\frac{1}{V} \sum_{k=1}^{n}\left(a_{k}\left|X_{k}-S_{k}^{0}\right| \sum_{j=1}^{n} \beta_{k j} c_{j} I_{j}\right) \\
& \leq \max _{0 \leq k, j \leq n}\left\{\frac{\beta_{k j} c_{j}}{a_{j}}\right\} \frac{1}{V} \sum_{k=1}^{n}\left(a_{k}\left|X_{k}-S_{k}^{0}\right| \sum_{j=1}^{n} a_{j} I_{j}\right) \\
& =\max _{0 \leq k, j \leq n}\left\{\frac{\beta_{k j} c_{j}}{a_{j}}\right\} \sum_{k=1}^{n}\left(a_{k}\left|X_{k}-S_{k}^{0}\right|\right) .
\end{aligned}
$$


In view of the definition of $a_{k}$ and (15), we have

$$
\begin{aligned}
& H_{2}=\frac{1}{V}\left[\sum_{k=1}^{n} \sum_{j=1}^{n} a_{k} \beta_{k j} c_{j} S_{k}^{0} I_{j}\right. \\
& \left.-\sum_{k=1}^{n} a_{k}\left(\epsilon_{k}+\gamma_{k}\right) I_{k}\right] \\
& =\frac{1}{V}\left[\sum_{k=1}^{n} \sum_{j=1}^{n} \omega_{k} \frac{\beta_{k j} c_{j} S_{k}^{0}}{\epsilon_{k}+\gamma_{k}} I_{j}-\sum_{k=1}^{n} \omega_{k} I_{k}\right] \\
& =\frac{1}{V}\left(\omega_{1}, \omega_{2}, \ldots, \omega_{n}\right) \\
& \times\left(M_{0}\left(I_{1}, I_{2}, \ldots, I_{n}\right)^{T}-\left(I_{1}, I_{2}, \ldots, I_{n}\right)^{T}\right) \\
& =\frac{1}{V}\left(\mathscr{R}_{0}-1\right) \sum_{k=1}^{n} \omega_{k} I_{k} \\
& =\frac{1}{V}\left(\mathscr{R}_{0}-1\right) \sum_{k=1}^{n}\left(\epsilon_{k}+\gamma_{k}\right) a_{k} I_{k} \\
& \leq \min _{1 \leq k \leq n}\left\{\epsilon_{k}+\gamma_{k}\right\}\left(\mathscr{R}_{0}-1\right) \mathbf{1}_{\mathscr{R}_{0} \leq 1} \\
& +\max _{1 \leq k \leq n}\left\{\epsilon_{k}+\gamma_{k}\right\}\left(\mathscr{R}_{0}-1\right) \mathbf{1}_{\mathscr{R}_{0} \geq 1}:=m_{1}, \\
& H_{3}=-\frac{\sum_{k=1}^{n} a_{k}^{2} \beta_{k}^{2} I_{k}^{2}}{2 V^{2}}=-\frac{\sum_{k=1}^{n} a_{k}^{2} \beta_{k}^{2} I_{k}^{2}}{2\left(\sum_{k=1}^{n}\left(a_{k} / \beta_{k}\right) \beta_{k} I_{k}\right)^{2}} \\
& \leq-\frac{\sum_{k=1}^{n} a_{k}^{2} \beta_{k}^{2} I_{k}^{2}}{2\left(\sum_{k=1}^{n} a_{k}^{2} \beta_{k}^{2} I_{k}^{2}\right)\left(\sum_{k=1}^{n}\left(1 / \beta_{k}^{2}\right)\right)} \\
& =-\frac{1}{2 \sum_{k=1}^{n}\left(1 / \beta_{k}^{2}\right)}:=m_{2} \text {. }
\end{aligned}
$$

Hence,

$$
\begin{aligned}
L(\log V) \leq & \max _{0 \leq k, j \leq n}\left\{\frac{\beta_{k j} c_{j}}{a_{j}}\right\} \\
& \times \sum_{k=1}^{n}\left(a_{k}\left|X_{k}-S_{k}^{0}\right|\right)+m_{1}+m_{2},
\end{aligned}
$$

which combined with (17) yields

$$
\begin{aligned}
\frac{\log V(t)}{t} \leq & \frac{\log V(0)}{t} \\
& +\max _{0 \leq k, j \leq n}\left\{\frac{\beta_{k j} c_{j}}{a_{j}}\right\} \sum_{k=1}^{n}\left(\frac{a_{k} \int_{0}^{t}\left|X_{k}-S_{k}^{0}\right| d t}{t}\right) \\
& +m_{1}+m_{2}-\sum_{k=1}^{n} a_{k} \beta_{k} \frac{\int_{0}^{t} I_{k} / V d B_{2 k}}{t} .
\end{aligned}
$$

By [22, Lemma 2.6], we get

$$
\lim _{t \rightarrow+\infty} \frac{\int_{0}^{t} I_{k} / V d B_{2 k}}{t}=0, \quad \text { a.s. }
$$

This together with (14) implies

$$
\begin{gathered}
\lim _{t \rightarrow \infty} \frac{\log V}{t} \leq \max _{0 \leq k, j \leq n}\left\{\frac{\beta_{k j} c_{j}}{a_{j}}\right\} \sum_{k=1}^{n} \frac{a_{k} \alpha_{k} S_{k}^{0}}{\sqrt{2 d_{k}-\alpha_{k}^{2}}} \\
+m_{1}+m_{2}, \quad \text { a.s. }
\end{gathered}
$$

Thus, the proof of Theorem 3 is completed.

Remark 4. From Theorem 3 we know that if $\mathscr{R}_{0}<1$ and $\alpha_{k}$, $1 \leq k \leq n$ are small, the disease will extinct exponentially; that is, $I_{k} \rightarrow 0$ as $t \rightarrow \infty$. By using the same arguments as in $[20,21]$, we get $S_{k}(t) \stackrel{w}{\rightarrow} v_{k}$ and $R_{k}(t) \rightarrow 0(t \rightarrow$ $+\infty)$, where $\stackrel{w}{\longrightarrow}$ means the weak convergence and $v_{k}$ is a distribution in $R_{+}$such that $\int_{0}^{\infty} x v_{k}(d x)=S_{k}^{0}$ and its density is $\left(M_{k} x^{2} p(x)\right)^{-1}$, where $M_{k}$ is a normal constant and $p(x)=x^{2 d_{k} / \alpha_{k}^{2}} \exp \left(2 d_{k} S_{k}^{0} / \alpha_{k}^{2} x\right), x>0$. That is, the solution of system (3) converges weakly to a singular measure (i.e., a distribution) when $\mathscr{R}_{0}<1$ and $\alpha_{k}, 1 \leq k \leq n$ are small.

Theorem 5. Assume $\left(C_{1}\right),\left(C_{2}\right)$ hold and $B=\left(\beta_{k j}\right)_{n \times n}$ is irreducible. Then the solution $Y(t)$ of system (3) with initial value $Y(0) \in R_{+}^{3 n}$ has the property $\lim _{t \rightarrow+\infty}\left(\log \left(\sum_{k=1}^{n} I_{k}\right) / t\right) \leq$ $c$, a.s. If $c<0$, the disease will extinct exponentially almost surely. Here $c:=\max _{1 \leq k, j \leq n}\left\{\beta_{k j} c_{j}\right\} \sum_{k=1}^{n} S_{k}^{0}-\min _{1 \leq k \leq n}\left\{\epsilon_{k}+\right.$ $\left.\gamma_{k}\right\}-1 / 2 \sum_{k=1}^{n}\left(1 / \beta_{k}^{2}\right)$.

Proof. Define $C^{2}$-function $V: R_{+}^{n} \rightarrow R_{+}$by $V\left(I_{1}, I_{2}, \ldots\right.$, $\left.I_{n}\right)=\sum_{k=1}^{n} I_{k}$. By Itô's formula, we compute

$$
d(\log V)=L(\log V) d t-\frac{1}{V} \sum_{k=1}^{n} \beta_{k} I_{k} d B_{2 k}
$$

where

$$
\begin{aligned}
L(\log V)= & \frac{1}{V} \sum_{k=1}^{n}\left[\sum_{j=1}^{n} \beta_{k j} S_{k} \phi_{j}\left(I_{j}\right)-\left(\epsilon_{k}+\gamma_{k}\right) I_{k}\right] \\
& -\frac{\sum_{k=1}^{n} \beta_{k}^{2} I_{k}^{2}}{2 V^{2}} \\
\leq & \frac{1}{V} \sum_{k=1}^{n}\left[\sum_{j=1}^{n} \beta_{k j} X_{k} c_{j} I_{j}-\left(\epsilon_{k}+\gamma_{k}\right) I_{k}\right] \\
& -\frac{\sum_{k=1}^{n} \beta_{k}^{2} I_{k}^{2}}{2\left(\sum_{k=1}^{n}\left(1 / \beta_{k}\right) \beta_{k} I_{k}\right)^{2}} \\
\leq & \max _{1 \leq k, j \leq n}\left\{\beta_{k j} c_{j}\right\} \sum_{k=1}^{n} X_{k} \\
& -\min _{1 \leq k \leq n}\left\{\epsilon_{k}+\gamma_{k}\right\}-\frac{1}{2 \sum_{k=1}^{n}\left(1 / \beta_{k}^{2}\right)} .
\end{aligned}
$$


Then

$$
\begin{aligned}
\frac{\log V(t)}{t} \leq & \frac{\log V(0)}{t}+\max _{1 \leq k, j \leq n}\left\{\beta_{k j} c_{j}\right\} \sum_{k=1}^{n} \frac{\int_{0}^{t} X_{k} d t}{t} \\
& -\min _{1 \leq k \leq n}\left\{\epsilon_{k}+\gamma_{k}\right\}-\frac{1}{2 \sum_{k=1}^{n}\left(1 / \beta_{k}^{2}\right)} \\
& -\sum_{k=1}^{n} \frac{\beta_{k} \int_{0}^{t} I_{k} / V d B_{2 k}}{t} .
\end{aligned}
$$

Taking $t \rightarrow \infty$, in view of (24) and (13), we get

$$
\begin{aligned}
\lim _{t \rightarrow+\infty} \frac{\log V(t)}{t} \leq & \max _{1 \leq k, j \leq n}\left\{\beta_{k j} c_{j}\right\} \sum_{k=1}^{n} S_{k}^{0} \\
& -\min _{1 \leq k \leq n}\left\{\epsilon_{k}+\gamma_{k}\right\}-\frac{1}{2 \sum_{k=1}^{n}\left(1 / \beta_{k}^{2}\right)}, \quad \text { a.s. }
\end{aligned}
$$

Remark 6. Theorem 5 tells us that the large perturbation forces the infective to expire regardless of the magnitude of $\mathscr{R}_{0}$.

\section{Ergodicity of System (3)}

When studying epidemic dynamical systems, we are also interested in when the disease will prevail and persist in a population. For a deterministic model, this is usually solved by showing that the endemic equilibrium is a global attractor or is globally asymptotically stable. But, for the stochastic system (3), there is no endemic equilibrium. In this section, we explore a weak stability. We show that there is a stationary distribution based on the theory of [23] (see Appendix), which reveals the disease will prevail.

From the proof of Theorem 2, we obtain $L V \leq \widetilde{M}$. Let $\widetilde{V}=$ $V+\widetilde{M}$. Then $L \widetilde{V} \leq \widetilde{V}$ and it is clear that $\inf _{Y \in R_{+}^{3 n} \backslash D_{k}} \widetilde{V}(Y) \rightarrow$ $\infty$ as $k \rightarrow \infty$, where $D_{k}=(1 / k, k) \times(1 / k, k) \times \cdots \times(1 / k, k)$. Hence, by Remark 2 of Theorem 4.1 of Hasminskii ([23], p. 86), we obtain that the solution $Y(t)$ is a homogeneous Markov process in $R_{+}^{3 n}$.

Theorem 7. Assume $\left(C_{1}\right)-\left(C_{4}\right)$ hold, $B=\left(\beta_{k j}\right)_{n \times n}$ is irreducible, and $\mathscr{R}_{0}=\rho\left(M_{0}\right)>1$. If $0<\alpha_{k}^{2}<d_{k}, 0<\beta_{k}^{2}<$ $\gamma_{k}+\epsilon_{k}, 0<\sigma_{k}^{2}<\delta_{k}, k=1,2, \ldots, n$ such that

$$
\begin{gathered}
\delta<\min \left\{\frac{\bar{c}_{k}}{2}\left(d_{k}-\alpha_{k}^{2}\right) S_{k}^{*}, \frac{b_{k}}{4}\left(\epsilon_{k}+\gamma_{k}-\beta_{k}^{2}\right)\left(I_{k}^{*}\right)^{2},\right. \\
\left.\frac{m_{k}}{2}\left(\delta_{k}-\sigma_{k}^{2}\right)\left(R_{k}^{*}\right)^{2}, k=1,2, \ldots, n\right\} .
\end{gathered}
$$

Then, for any initial value $Y(0) \in R_{+}^{3 n}$, there is a stationary distribution $\mu(\cdot)$ for system (3) and it has ergodic property, where $M_{0}=M\left(S^{0}\right)=\left(\beta_{k j} S_{k}^{0} c_{j} /\left(\epsilon_{k}+\gamma_{k}\right)\right)_{n \times n}, S_{k}^{0}=\Lambda_{k} / d_{k}$,

$$
\begin{aligned}
\delta=\sum_{k=1}^{n}[ & \left(\frac{a+2}{2} \bar{c}_{k}+b_{k} S_{k}^{*}\right) S_{k}^{*} \alpha_{k}^{2} \\
& \left.+\left(\frac{a+1}{2} \bar{c}_{k}+b_{k} I_{k}^{*}\right) I_{k}^{*} \beta_{k}^{2}+m_{k}\left(R_{k}^{*}\right)^{2} \sigma_{k}^{2}\right],
\end{aligned}
$$

$a, b_{k}, m_{k}, k=1,2, \ldots, n$ are defined as in the proof, $P^{*}=$ $\left(S_{1}^{*}, I_{1}^{*}, R_{1}^{*}, \ldots, S_{n}^{*}, I_{n}^{*}, R_{n}^{*}\right)$ is the endemic equilibrium of system (1), and $\bar{c}_{k}, k=1,2, \ldots, n$ denote the cofactor of the $k t h$ diagonal element of $L_{\bar{B}}, \bar{B}=\left(\bar{\beta}_{k j}\right)_{n \times n}=\left(\beta_{k j} S_{k}^{*} \phi_{j}\left(I_{j}^{*}\right)\right)_{n \times n}$.

Proof. Since $\mathscr{R}_{0}>1$ and $\left(C_{1}\right)-\left(C_{4}\right)$ hold, from Proposition 1 there is an endemic equilibrium $P^{*}$ of system (1). Then

$$
\begin{aligned}
& \sum_{j=1}^{n} \beta_{k j} S_{k}^{*} \phi_{j}\left(I_{j}^{*}\right)+d_{k} S_{k}^{*}=\Lambda_{k}, \\
& \sum_{j=1}^{n} \beta_{k j} S_{k}^{*} \phi_{j}\left(I_{j}^{*}\right)=\left(\epsilon_{k}+\gamma_{k}\right) I_{k}^{*} .
\end{aligned}
$$

Define

$$
\begin{aligned}
V(Y)= & a \sum_{k=1}^{n} \bar{c}_{k}\left(S_{k}-S_{k}^{*}-S_{k}^{*} \log \frac{S_{k}}{S_{k}^{*}}+I_{k}-I_{k}^{*}-I_{k}^{*} \log \frac{I_{k}}{I_{k}^{*}}\right) \\
& +\sum_{k=1}^{n} \bar{c}_{k}\left(I_{k}-I_{k}^{*}-\log \frac{I_{k}}{I_{k}^{*}}\right) \\
& +\frac{1}{2} \sum_{k=1}^{n} b_{k}\left(S_{k}-S_{k}^{*}+I_{k}-I_{k}^{*}\right)^{2} \\
& +\frac{1}{2} \sum_{k=1}^{n} l_{k}\left(S_{k}-S_{k}^{*}\right)^{2}+\frac{1}{2} \sum_{k=1}^{n} m_{k}\left(R_{k}-R_{k}^{*}\right)^{2} \\
:= & a V_{1}+V_{2}+V_{3}+V_{4}+V_{5},
\end{aligned}
$$

where $a, b_{k}, m_{k}, l_{k}, k=1,2, \ldots, n$ are positive constants to be determined later, and $\bar{c}_{k}>0, k=1,2, \ldots, n$ according to Lemma A.1. Then $V$ is positive definite. In view of (32) and the inequality $(x+y)^{2} \leq 2\left(x^{2}+y^{2}\right)$, by direct calculation, we get $L V_{i}(i=1,2, \ldots, 5)$ as follows:

$$
\begin{aligned}
L V_{1}= & \sum_{k=1}^{n} \bar{c}_{k}\left(1-\frac{S_{k}^{*}}{S_{k}}\right)\left(\Lambda_{k}-\sum_{j=1}^{n} \beta_{k j} S_{k} \phi_{j}\left(I_{j}\right)-d_{k} S_{k}\right) \\
& +\sum_{k=1}^{n} \frac{\bar{c}_{k} S_{k}^{*} \alpha_{k}^{2}}{2} \\
& +\sum_{k=1}^{n} \bar{c}_{k}\left(1-\frac{I_{k}^{*}}{I_{k}}\right)\left(\sum_{j=1}^{n} \beta_{k j} S_{k} \phi_{j}\left(I_{j}\right)-\left(\epsilon_{k}+\gamma_{k}\right) I_{k}\right) \\
& +\sum_{k=1}^{n} \frac{\bar{c}_{k} I_{k}^{*} \beta_{k}^{2}}{2}
\end{aligned}
$$




$$
\begin{aligned}
= & \sum_{k=1}^{n} \bar{c}_{k}\left(1-\frac{S_{k}^{*}}{S_{k}}\right) \\
& \times\left(\sum_{j=1}^{n} \bar{\beta}_{k j}\left(1-\frac{S_{k} \phi_{j}\left(I_{j}\right)}{S_{k}^{*} \phi_{j}\left(I_{j}^{*}\right)}\right)-d_{k}\left(S_{k}-S_{k}^{*}\right)\right) \\
& +\sum_{k=1}^{n} \frac{\bar{c}_{k} S_{k}^{*} \alpha_{k}^{2}}{2} \\
& +\sum_{k=1}^{n} \bar{c}_{k}\left(1-\frac{I_{k}^{*}}{I_{k}}\right) \\
& \times\left(\sum_{j=1}^{n} \bar{\beta}_{k j} \frac{S_{k} \phi_{j}\left(I_{j}\right)}{S_{k}^{*} \phi_{j}\left(I_{j}^{*}\right)}-\sum_{j=1}^{n} \bar{\beta}_{k j} \frac{I_{k}}{I_{k}^{*}}\right) \\
& +\sum_{k=1}^{n} \frac{\bar{c}_{k} I_{k}^{*} \beta_{k}^{2}}{2} \\
= & \sum_{k=1}^{n} \bar{c}_{k}\left[\sum_{j=1}^{n} \bar{\beta}_{k j}\left(1-\frac{S_{k}^{*}}{S_{k}}+\frac{\phi_{j}\left(I_{j}\right)}{\phi_{j}\left(I_{j}^{*}\right)}-\frac{S_{k} \phi_{j}\left(I_{j}\right)}{S_{k}^{*} \phi_{j}\left(I_{j}^{*}\right)}\right)\right. \\
& +\sum_{k=1}^{n} \frac{\sum_{k} \bar{c}_{k}}{2}\left[-\frac{S_{k} \phi_{j}\left(I_{j}\right) I_{k}^{*}}{S_{k}^{*} \phi_{j}\left(I_{j}^{*}\right) I_{k}}\right) \\
& \left.+\sum_{k=1}^{2} \frac{\left(S_{k}\right.}{S_{k}} \frac{\left(S_{k}-S_{k}^{*}\right)^{2}}{S_{k}}\right]+\sum_{k=1}^{n} \frac{S_{k}^{*} \bar{c}_{k} S_{k}^{*} \alpha_{k}^{2}}{2} \\
& {\left[\sum _ { j = 1 } ^ { n } \overline { \beta } _ { k j } \left(1-\frac{I_{k}^{*} \phi_{j}}{I_{k}^{*}}+\frac{\left.S_{k} \phi_{j}\left(I_{j}^{*}\right) I_{j}\right)}{S_{k}^{*} \phi_{j}\left(I_{j}^{*}\right)}\right.\right.}
\end{aligned}
$$

From the calculation of $L V_{1}$, we directly get

$$
\begin{aligned}
L V_{2}= & \sum_{k=1}^{n} \bar{c}_{k}\left[\sum_{j=1}^{n} \bar{\beta}_{k j}\left(1-\frac{I_{k}}{I_{k}^{*}}+\frac{S_{k} \phi_{j}\left(I_{j}\right)}{S_{k}^{*} \phi_{j}\left(I_{j}^{*}\right)}-\frac{S_{k} \phi_{j}\left(I_{j}\right) I_{k}^{*}}{S_{k}^{*} \phi_{j}\left(I_{j}^{*}\right) I_{k}}\right)\right] \\
& +\sum_{k=1}^{n} \frac{\bar{c}_{k} I_{k}^{*} \beta_{k}^{2}}{2}
\end{aligned}
$$

$$
\begin{aligned}
& L V_{3}=\sum_{k=1}^{n} b_{k}\left\{\left(S_{k}-S_{k}^{*}+I_{k}-I_{k}^{*}\right)\right. \\
& \times\left[\Lambda_{k}-d_{k} S_{k}-\left(\epsilon_{k}+\gamma_{k}\right) I_{k}\right] \\
& \left.+\frac{S_{k}^{2} \alpha_{k}^{2}}{2}+\frac{I_{k}^{2} \beta_{k}^{2}}{2}\right\} \\
& =\sum_{k=1}^{n} b_{k}\left\{\left(S_{k}-S_{k}^{*}+I_{k}-I_{k}^{*}\right)\right. \\
& \times\left[-d_{k}\left(S_{k}-S_{k}^{*}\right)-\left(\epsilon_{k}+\gamma_{k}\right)\left(I_{k}-I_{k}^{*}\right)\right] \\
& \left.+\frac{S_{k}^{2} \alpha_{k}^{2}}{2}+\frac{I_{k}^{2} \beta_{k}^{2}}{2}\right\} \\
& \leq-\sum_{k=1}^{n} b_{k}\left[d_{k}\left(S_{k}-S_{k}^{*}\right)^{2}+\left(\epsilon_{k}+\gamma_{k}\right)\left(I_{k}-I_{k}^{*}\right)^{2}\right. \\
& \left.-\left(\gamma_{k}+d_{k}+\epsilon_{k}\right)\left(S_{k}-S_{k}^{*}\right)\left(I_{k}-I_{k}^{*}\right)\right] \\
& +\sum_{k=1}^{n} b_{k} \alpha_{k}^{2}\left[\left(S_{k}-S_{k}^{*}\right)^{2}+\left(S_{k}^{*}\right)^{2}\right] \\
& +\sum_{k=1}^{n} b_{k} \beta_{k}^{2}\left[\left(I_{k}-I_{k}^{*}\right)^{2}+\left(I_{k}^{*}\right)^{2}\right] \\
& =-\sum_{k=1}^{n} b_{k}\left[\left(d_{k}-\alpha_{k}^{2}\right)\left(S_{k}-S_{k}^{*}\right)^{2}\right. \\
& \left.+\left(\epsilon_{k}+\gamma_{k}-\beta_{k}^{2}\right)\left(I_{k}-I_{k}^{*}\right)^{2}\right] \\
& -\sum_{k=1}^{n} b_{k}\left(\gamma_{k}+d_{k}+\epsilon_{k}\right)\left(S_{k}-S_{k}^{*}\right)\left(I_{k}-I_{k}^{*}\right) \\
& +\sum_{k=1}^{n} b_{k}\left[\alpha_{k}^{2}\left(S_{k}^{*}\right)^{2}+\beta_{k}^{2}\left(I_{k}^{*}\right)^{2}\right] \\
& \leq-\sum_{k=1}^{n} b_{k}\left[\left(d_{k}-\alpha_{k}^{2}-\frac{\left(d_{k}+\gamma_{k}+\epsilon_{k}\right)^{2}}{2\left(\gamma_{k}+d_{k}+\epsilon_{k}-\beta_{k}^{2}\right)}\right)\right. \\
& \left.\times\left(S_{k}-S_{k}^{*}\right)^{2}+\frac{1}{2}\left(\epsilon_{k}+\gamma_{k}-\beta_{k}^{2}\right)\left(I_{k}-I_{k}^{*}\right)^{2}\right] \\
& +\sum_{k=1}^{n} b_{k}\left[\alpha_{k}^{2}\left(S_{k}^{*}\right)^{2}+\beta_{k}^{2}\left(I_{k}^{*}\right)^{2}\right]
\end{aligned}
$$$$
L V_{4}=\sum_{k=1}^{n} l_{k}\left(S_{k}-S_{k}^{*}\right)
$$$$
\times\left(\Lambda_{k}-\sum_{j=1}^{n} \beta_{k j} S_{k} \phi_{j}\left(I_{j}\right)-d_{k} S_{k}\right)
$$$$
+\sum_{k=1}^{n} \frac{l_{k} S_{k}^{2} \alpha_{k}^{2}}{2}
$$ 


$$
\begin{aligned}
& =\sum_{k=1}^{n} l_{k}\left(S_{k}-S_{k}^{*}\right) \\
& \times\left(\sum_{j=1}^{n} \beta_{k j}\left(S_{k}^{*} \phi_{j}\left(I_{j}^{*}\right)-S_{k} \phi_{j}\left(I_{j}\right)\right)\right. \\
& \left.-d_{k}\left(S_{k}-S_{k}^{*}\right)\right) \\
& +\sum_{k=1}^{n} \frac{l_{k} S_{k}^{2} \alpha_{k}^{2}}{2} \\
& =-\sum_{k=1}^{n} \sum_{j=1}^{n} l_{k} \beta_{k j} S_{k}^{*} \\
& \times\left(S_{k}-S_{k}^{*}\right)\left(\phi_{j}\left(I_{j}\right)-\phi_{j}\left(I_{j}^{*}\right)\right) \\
& -\sum_{k=1}^{n} \sum_{j=1}^{n} l_{k} \beta_{k j}\left(S_{k}-S_{k}^{*}\right)^{2} \phi_{j}\left(I_{j}\right) \\
& -\sum_{k=1}^{n} l_{k} d_{k}\left(S_{k}-S_{k}^{*}\right)^{2}+\sum_{k=1}^{n} \frac{l_{k} S_{k}^{2} \alpha_{k}^{2}}{2} \\
& \leq-\sum_{k=1}^{n} \sum_{j=1}^{n} l_{k} \beta_{k j} S_{k}^{*}\left(S_{k}-S_{k}^{*}\right)\left(\phi_{j}\left(I_{j}\right)-\phi_{j}\left(I_{j}^{*}\right)\right) \\
& -\sum_{k=1}^{n} l_{k}\left(d_{k}-\alpha_{k}^{2}\right)\left(S_{k}-S_{k}^{*}\right)^{2} \\
& +\sum_{k=1}^{n} l_{k}\left(S_{k}^{*}\right)^{2} \alpha_{k}^{2} \\
& L V_{5}=\sum_{k=1}^{n} m_{k}\left(R_{k}-R_{k}^{*}\right)\left(\gamma_{k} I_{k}-\delta_{k} R_{k}\right) \\
& +\sum_{k=1}^{n} \frac{m_{k} R_{k}^{2} \sigma_{k}^{2}}{2} \\
& =\sum_{k=1}^{n} m_{k}\left(R_{k}-R_{k}^{*}\right) \\
& \times\left[\gamma_{k}\left(I_{k}-I_{k}^{*}\right)-\delta_{k}\left(R_{k}-R_{k}^{*}\right)\right] \\
& +\sum_{k=1}^{n} \frac{m_{k} R_{k}^{2} \sigma_{k}^{2}}{2} \\
& \leq \sum_{k=1}^{n} m_{k} \gamma_{k}\left(I_{k}-I_{k}^{*}\right)\left(R_{k}-R_{k}^{*}\right) \\
& -\sum_{k=1}^{n} m_{k}\left(\delta_{k}-\sigma_{k}^{2}\right)\left(R_{k}-R_{k}^{*}\right)^{2} \\
& +\sum_{k=1}^{n} m_{k}\left(R_{k}^{*}\right)^{2} \sigma_{k}^{2}
\end{aligned}
$$$$
\leq \sum_{k=1}^{n} \frac{m_{k} \gamma_{k}^{2}}{2\left(\delta_{k}-\sigma_{k}^{2}\right)}\left(I_{k}-I_{k}^{*}\right)^{2}
$$

By Lemma A.3, we know

$$
\begin{gathered}
\sum_{k=1}^{n} \bar{c}_{k}\left(\sum_{j=1}^{n} \bar{\beta}_{k j} \frac{\phi_{j}\left(I_{j}\right)}{\phi_{j}\left(I_{j}^{*}\right)}-\sum_{j=1}^{n} \bar{\beta}_{k j} \frac{\phi_{k}\left(I_{k}\right)}{\phi_{k}\left(I_{k}^{*}\right)}\right)=0, \\
\sum_{k=1}^{n} \bar{c}_{k}\left(\sum_{j=1}^{n} \bar{\beta}_{k j} \log \frac{\phi_{j}\left(I_{j}\right)}{\phi_{j}\left(I_{j}^{*}\right)}-\sum_{j=1}^{n} \bar{\beta}_{k j} \log \frac{\phi_{k}\left(I_{k}\right)}{\phi_{k}\left(I_{k}^{*}\right)}\right)=0 .
\end{gathered}
$$

Besides note that $a-1-\log a \geq 0$ for $a>0$; then

$$
\begin{gathered}
1-\frac{S_{k}^{*}}{S_{k}} \leq-\log \frac{S_{k}^{*}}{S_{k}} \\
1-\frac{S_{k} \phi_{j}\left(I_{j}\right) I_{k}^{*}}{S_{k}^{*} \phi_{j}\left(I_{j}^{*}\right) I_{k}} \leq-\log \frac{S_{k} \phi_{j}\left(I_{j}\right) I_{k}^{*}}{S_{k}^{*} \phi_{j}\left(I_{j}^{*}\right) I_{k}} \\
=-\log \frac{S_{k}}{S_{k}^{*}}-\log \frac{\phi_{j}\left(I_{j}\right)}{\phi_{j}\left(I_{j}^{*}\right)}-\log \frac{I_{k}^{*}}{I_{k}} \\
-\log \frac{\phi_{k}\left(I_{k}\right)}{\phi_{k}\left(I_{k}^{*}\right)}-\log \frac{I_{k}^{*}}{I_{k}}=-\log \frac{\phi_{k}\left(I_{k}\right) I_{k}^{*}}{\phi_{k}\left(I_{k}^{*}\right) I_{k}} \leq \frac{\phi_{k}\left(I_{k}^{*}\right) I_{k}}{\phi_{k}\left(I_{k}\right) I_{k}^{*}}-1 .
\end{gathered}
$$

Successively substituting (39), (40), (37), (38), and (41) into (34) yields

$$
\begin{aligned}
& L V_{1} \leq-\sum_{k=1}^{n} \frac{\bar{c}_{k} d_{k}\left(S_{k}-S_{k}^{*}\right)^{2}}{S_{k}} \\
&+\sum_{k=1}^{n} \bar{c}_{k} \sum_{j=1}^{n} \bar{\beta}_{k j}\left(-\log \frac{S_{k}^{*}}{S_{k}}-\log \frac{S_{k}}{S_{k}^{*}}\right. \\
&-\log \frac{\phi_{j}\left(I_{j}\right)}{\phi_{j}\left(I_{j}^{*}\right)}-\log \frac{I_{k}^{*}}{I_{k}} \\
&\left.-\frac{I_{k}}{I_{k}^{*}}+\frac{\phi_{j}\left(I_{j}\right)}{\phi_{j}\left(I_{j}^{*}\right)}\right)
\end{aligned}
$$




$$
\begin{aligned}
& +\sum_{k=1}^{n} \bar{c}_{k}\left[\frac{S_{k}^{*} \alpha_{k}^{2}}{2}+\frac{I_{k}^{*} \beta_{k}^{2}}{2}\right] \\
= & -\sum_{k=1}^{n} \frac{\bar{c}_{k} d_{k}\left(S_{k}-S_{k}^{*}\right)^{2}}{S_{k}} \\
& +\sum_{k=1}^{n} \bar{c}_{k} \sum_{j=1}^{n} \bar{\beta}_{k j}\left(-\log \frac{\phi_{k}\left(I_{k}\right)}{\phi_{k}\left(I_{k}^{*}\right)}\right. \\
& +\sum_{k=1}^{n} \bar{c}_{k}\left[\frac{S_{k}^{*} \alpha_{k}^{2}}{2}+\frac{I_{k}^{*} \beta_{k}^{2}}{2}\right] \\
\leq & \left.-\sum_{k=1}^{n} \frac{I_{k}^{*}}{I_{k}}-\frac{I_{k}}{I_{k}^{*}}+\frac{\phi_{k}\left(I_{k}\right)}{\phi_{k}\left(I_{k}^{*}\right)}\right) \\
& +\sum_{k=1}^{n} \bar{c}_{k} \sum_{j=1}^{n} \bar{\beta}_{k j}\left(\frac{\left.S_{k}^{*}\right)^{2}}{\phi_{k}\left(I_{k}^{*}\right) I_{k}^{*}}-1-\frac{I_{k}}{I_{k}^{*}}+\frac{\phi_{k}\left(I_{k}\right)}{\phi_{k}\left(I_{k}^{*}\right)}\right) \\
& +\sum_{k=1}^{n} \bar{c}_{k}\left[\frac{S_{k}^{*} \alpha_{k}^{2}}{2}+\frac{I_{k}^{*} \beta_{k}^{2}}{2}\right] .
\end{aligned}
$$

In view of $\left(C_{4}\right)$, we obtain

$$
\begin{aligned}
\frac{\phi_{k}\left(I_{k}^{*}\right) I_{k}}{\phi_{k}\left(I_{k}\right) I_{k}^{*}}-1-\frac{I_{k}}{I_{k}^{*}}+\frac{\phi_{k}\left(I_{k}\right)}{\phi_{k}\left(I_{k}^{*}\right)} \\
=\frac{1}{\phi_{k}\left(I_{k}^{*}\right)} \frac{I_{k}}{\phi_{k}\left(I_{k}\right)}\left(\phi_{k}\left(I_{k}^{*}\right)-\phi_{k}\left(I_{k}\right)\right) \\
\quad \times\left(\frac{\phi_{k}\left(I_{k}^{*}\right)}{I_{k}^{*}}-\frac{\phi_{k}\left(I_{k}\right)}{I_{k}}\right) \leq 0 .
\end{aligned}
$$

Then

$$
\begin{aligned}
L V_{1} \leq & -\sum_{k=1}^{n} \frac{\bar{c}_{k} d_{k}\left(S_{k}-S_{k}^{*}\right)^{2}}{S_{k}} \\
& +\sum_{k=1}^{n} \bar{c}_{k}\left[\frac{S_{k}^{*} \alpha_{k}^{2}}{2}+\frac{I_{k}^{*} \beta_{k}^{2}}{2}\right] .
\end{aligned}
$$

By using inequality $a-1-\log a \geq 0$ for $a>0$ again, it follows that

$$
-\log \frac{S_{k}}{S_{k}^{*}} \leq \frac{S_{k}^{*}}{S_{k}}-1 .
$$

Successively substituting (40), (37), (45), (41), and (43) into (35), we obtain

$$
\begin{array}{r}
L V_{2} \leq \sum_{k=1}^{n} \bar{c}_{k}\left[\sum _ { j = 1 } ^ { n } \overline { \beta } _ { k j } \left(-\frac{I_{k}}{I_{k}^{*}}+\frac{S_{k} \phi_{j}\left(I_{j}\right)}{S_{k}^{*} \phi_{j}\left(I_{j}^{*}\right)}-\log \frac{S_{k}}{S_{k}^{*}}\right.\right. \\
\left.\left.\quad-\log \frac{\phi_{j}\left(I_{j}\right)}{\phi_{j}\left(I_{j}^{*}\right)}-\log \frac{I_{k}^{*}}{I_{k}}\right)\right] \\
+\sum_{k=1}^{n} \frac{\bar{c}_{k} I_{k}^{*} \beta_{k}^{2}}{2} \\
=\sum_{k=1}^{n} \bar{c}_{k}\left[\sum _ { j = 1 } ^ { n } \overline { \beta } _ { k j } \left(-\frac{I_{k}}{I_{k}^{*}}+\frac{S_{k} \phi_{j}\left(I_{j}\right)}{S_{k}^{*} \phi_{j}\left(I_{j}^{*}\right)}-\log \frac{S_{k}}{S_{k}^{*}}\right.\right. \\
\left.\left.\quad+\sum_{k=1}^{n} \frac{\bar{c}_{k} I_{k}^{*} \beta_{k}^{2}}{2} \frac{\phi_{k}\left(I_{k}\right)}{\phi_{k}\left(I_{k}^{*}\right)}-\log \frac{I_{k}^{*}}{I_{k}}\right)\right] \\
\leq \sum_{k=1}^{n} \bar{c}_{k}\left[\sum _ { j = 1 } ^ { n } \overline { \beta } _ { k j } \left(-\frac{I_{k}}{I_{k}^{*}}+\frac{S_{k} \phi_{j}\left(I_{j}\right)}{S_{k}^{*} \phi_{j}\left(I_{j}^{*}\right)}+\frac{S_{k}^{*}}{S_{k}}\right.\right.
\end{array}
$$

$$
\left.\left.-1+\frac{I_{k} \phi_{k}\left(I_{k}^{*}\right)}{I_{k}^{*} \phi_{k}\left(I_{k}\right)}-1\right)\right]
$$

$+\sum_{k=1}^{n} \frac{\bar{c}_{k} I_{k}^{*} \beta_{k}^{2}}{2}$

$=\sum_{k=1}^{n} \bar{c}_{k}\left(\sum_{j=1}^{n} \bar{\beta}_{k j}\left[\left(\frac{S_{k}}{S_{k}^{*}}-1\right)\left(\frac{\phi_{j}\left(I_{j}\right)}{\phi_{j}\left(I_{j}^{*}\right)}-1\right)\right.\right.$

$$
+\left(\frac{S_{k}}{S_{k}^{*}}+\frac{S_{k}^{*}}{S_{k}}-2\right)
$$

$$
+\left(\frac{\phi_{k}\left(I_{k}^{*}\right) I_{k}}{\phi_{k}\left(I_{k}\right) I_{k}^{*}}-1-\frac{I_{k}}{I_{k}^{*}}\right.
$$

$$
\left.\left.\left.+\frac{\phi_{k}\left(I_{k}\right)}{\phi_{k}\left(I_{k}^{*}\right)}\right)\right]\right)
$$

$$
\begin{aligned}
+\sum_{k=1}^{n} \frac{\bar{c}_{k} I_{k}^{*} \beta_{k}^{2}}{2} & \\
\leq \sum_{k=1}^{n} \bar{c}_{k}\left(\sum_{j=1}^{n} \bar{\beta}_{k j}[\right. & \left(\frac{S_{k}}{S_{k}^{*}}-1\right)\left(\frac{\phi_{j}\left(I_{j}\right)}{\phi_{j}\left(I_{j}^{*}\right)}-1\right) \\
& \left.\left.+\left(\frac{S_{k}}{S_{k}^{*}}+\frac{S_{k}^{*}}{S_{k}}-2\right)\right]\right)
\end{aligned}
$$




$$
\begin{aligned}
& +\sum_{k=1}^{n} \frac{\bar{c}_{k} I_{k}^{*} \beta_{k}^{2}}{2} \\
& =\sum_{k=1}^{n} \bar{c}_{k}\left(\sum_{j=1}^{n} \beta_{k j}\left(S_{k}-S_{k}^{*}\right)\left(\phi_{j}\left(I_{j}\right)-\phi_{j}\left(I_{j}^{*}\right)\right)\right. \\
& \left.\quad+\sum_{j=1}^{n} \beta_{k j} \phi_{j}\left(I_{j}^{*}\right) \frac{\left(S_{k}-S_{k}^{*}\right)^{2}}{S_{k}}\right) \\
& +\sum_{k=1}^{n} \frac{\bar{c}_{k} I_{k}^{*} \beta_{k}^{2}}{2} .
\end{aligned}
$$

Hence

$$
\begin{aligned}
a L V_{1}+ & L V_{2}+L V_{4} \\
\leq & -\sum_{k=1}^{n}\left[\sum_{j=1}^{n} \beta_{k j}\left(l_{k} S_{k}^{*}-\bar{c}_{k}\right)\left(S_{k}-S_{k}^{*}\right)\left(\phi_{j}\left(I_{j}\right)-\phi_{j}\left(I_{j}^{*}\right)\right)\right. \\
& \left.+\bar{c}_{k}\left(a d_{k}-\sum_{j=1}^{n} \beta_{k j} \phi_{j}\left(I_{j}^{*}\right)\right) \frac{\left(S_{k}-S_{k}^{*}\right)^{2}}{S_{k}}\right] \\
& -\sum_{k=1}^{n} l_{k}\left(d_{k}-\alpha_{k}^{2}\right)\left(S_{k}-S_{k}^{*}\right)^{2} \\
+ & \sum_{k=1}^{n} \bar{c}_{k}\left[\frac{\beta_{k}^{2}}{2}(a+1) I_{k}^{*}+\frac{\alpha_{k}^{2}}{2}(a+2) S_{k}^{*}\right] .
\end{aligned}
$$

Choose $l_{k}=\bar{c}_{k} / S_{k}^{*}$ and $a=\max \left\{\sum_{j=1}^{n} \beta_{k j} I_{j}^{*} / d_{k}, k=\right.$ $1,2, \ldots, n\}$ such that $l_{k} S_{k}^{*}-\bar{c}_{k}=0$ and $a d_{k}-\sum_{j=1}^{n} \beta_{k j} I_{j}^{*} \geq$ $0, k=1,2, \ldots, n$. Then

$$
\begin{aligned}
a L V_{1}+ & L V_{2}+L V_{4} \\
\leq & -\sum_{k=1}^{n} \frac{\bar{c}_{k}}{S_{k}^{*}}\left(d_{k}-\alpha_{k}^{2}\right)\left(S_{k}-S_{k}^{*}\right)^{2} \\
& +\sum_{k=1}^{n} \bar{c}_{k}\left[\frac{\beta_{k}^{2}}{2}(a+1) I_{k}^{*}+\frac{\alpha_{k}^{2}}{2}(a+2) S_{k}^{*}\right] .
\end{aligned}
$$

Furthermore,

$$
\begin{aligned}
& a L V_{1}+ L V_{2}+L V_{3}+L V_{4}+L V_{5} \\
& \leq- \sum_{k=1}^{n}\left[b_{k}\left(d_{k}-\alpha_{k}^{2}-\frac{\left(d_{k}+\gamma_{k}+\epsilon_{k}\right)^{2}}{2\left(\gamma_{k}+d_{k}+\epsilon_{k}-\beta_{k}^{2}\right)}\right)\right. \\
&\left.+\frac{\bar{c}_{k}}{S_{k}^{*}}\left(d_{k}-\alpha_{k}^{2}\right)\right]\left(S_{k}-S_{k}^{*}\right)^{2} \\
&-\frac{1}{2} \sum_{k=1}^{n}\left(b_{k}\left(\epsilon_{k}+\gamma_{k}-\beta_{k}^{2}\right)-\frac{m_{k} \gamma_{k}^{2}}{\delta_{k}-\sigma_{k}^{2}}\right)\left(I_{k}-I_{k}^{*}\right)^{2}
\end{aligned}
$$

$$
\begin{aligned}
& -\frac{1}{2} \sum_{k=1}^{n} m_{k}\left(\delta_{k}-\sigma_{k}^{2}\right)\left(R_{k}-R_{k}^{*}\right)^{2} \\
& +\sum_{k=1}^{n}\left[\left(\frac{a+2}{2} \bar{c}_{k}+b_{k} S_{k}^{*}\right) S_{k}^{*} \alpha_{k}^{2}+\left(\frac{a+1}{2} \bar{c}_{k}+b_{k} I_{k}^{*}\right)\right. \\
& \left.\times I_{k}^{*} \beta_{k}^{2}+m_{k}\left(R_{k}^{*}\right)^{2} \sigma_{k}^{2}\right] .
\end{aligned}
$$

Choose $b_{k}>0$ such that $b_{k}\left(d_{k}-\alpha_{k}^{2}-\left(d_{k}+\gamma_{k}+\epsilon_{k}\right)^{2} / 2\left(\gamma_{k}+\right.\right.$ $\left.\left.d_{k}+\epsilon_{k}-\beta_{k}^{2}\right)\right)+\left(\bar{c}_{k} / S_{k}^{*}\right)\left(d_{k}-\alpha_{k}^{2}\right) \geq\left(\bar{c}_{k} / 2 S_{k}^{*}\right)\left(d_{k}-\alpha_{k}^{2}\right)$ and choose $m_{k}=\left(b_{k} / 2\right)\left(\epsilon_{k}+\gamma_{k}-\beta_{k}^{2}\right)\left(\left(\delta_{k}-\sigma_{k}^{2}\right) / \gamma_{k}^{2}\right)$ such that $b_{k}\left(\epsilon_{k}+\gamma_{k}-\beta_{k}^{2}\right)-m_{k} \gamma_{k}^{2} /\left(\delta_{k}-\sigma_{k}^{2}\right)=\left(b_{k} / 2\right)\left(\epsilon_{k}+\gamma_{k}-\beta_{k}^{2}\right)$; then

$$
\begin{aligned}
a L V_{1}+ & L V_{2}+L V_{3}+L V_{4}+L V_{5} \\
\leq & -\sum_{k=1}^{n} \frac{\bar{c}_{k}}{2 S_{k}^{*}}\left(d_{k}-\alpha_{k}^{2}\right)\left(S_{k}-S_{k}^{*}\right)^{2} \\
& -\frac{1}{4} \sum_{k=1}^{n} b_{k}\left(\epsilon_{k}+\gamma_{k}-\beta_{k}^{2}\right)\left(I_{k}-I_{k}^{*}\right)^{2} \\
& -\frac{1}{2} \sum_{k=1}^{n} m_{k}\left(\delta_{k}-\sigma_{k}^{2}\right)\left(R_{k}-R_{k}^{*}\right)^{2} \\
& +\sum_{k=1}^{n}\left[\left(\frac{a+2}{2} \bar{c}_{k}+b_{k} S_{k}^{*}\right) S_{k}^{*} \alpha_{k}^{2}\right. \\
& +\sum_{k=1}^{n} \frac{m_{k}}{2}\left(\delta_{k}-\sigma_{k}^{2}\right)\left(R_{k}-R_{k}^{*}\right)^{2}+\delta . \\
& +\sum_{k=1}^{n} \frac{b_{k}}{4}\left(\epsilon_{k}+\gamma_{k}-\beta_{k}^{2}\right)\left(I_{k}-I_{k}^{*}\right)^{2} \\
:= & -\sum_{k=1}^{n} \frac{\bar{c}_{k}}{2 S_{k}^{*}}\left(d_{k}-\alpha_{k}^{2}\right)\left(S_{k}^{*}\right)_{k}^{2} \sigma_{k}^{2} \beta_{k}^{2} \\
& \left.+S_{k}^{*}\right)^{2} \\
& \\
& \\
& \\
&
\end{aligned}
$$

Note that

$$
\begin{gathered}
\delta<\min \left\{\frac{\bar{c}_{k}}{2}\left(d_{k}-\alpha_{k}^{2}\right) S_{k}^{*}, \frac{b_{k}}{4}\left(\epsilon_{k}+\gamma_{k}-\beta_{k}^{2}\right)\left(I_{k}^{*}\right)^{2},\right. \\
\left.\frac{m_{k}}{2}\left(\delta_{k}-\sigma_{k}^{2}\right)\left(R_{k}^{*}\right)^{2}, k=1,2, \ldots, n\right\} .
\end{gathered}
$$


Then the ellipsoid

$$
\begin{aligned}
& -\sum_{k=1}^{n} \frac{\bar{c}_{k}}{2 S_{k}^{*}}\left(d_{k}-\alpha_{k}^{2}\right)\left(S_{k}-S_{k}^{*}\right)^{2} \\
& -\sum_{k=1}^{n} \frac{b_{k}}{4}\left(\epsilon_{k}+\gamma_{k}-\beta_{k}^{2}\right)\left(I_{k}-I_{k}^{*}\right)^{2} \\
& -\sum_{k=1}^{n} \frac{m_{k}}{2}\left(\delta_{k}-\sigma_{k}^{2}\right)\left(R_{k}-R_{k}^{*}\right)^{2}+\delta=0
\end{aligned}
$$

lies entirely in $R_{+}^{3 n}$. We can take $U$ to be a neighborhood of the ellipsoid with $\bar{U} \subseteq E_{3 n}=R_{+}^{3 n}$, so for $x \in E_{3 n} \backslash U, L V \leq$ $-K$ ( $K$ is a positive constant), which implies condition (B.2) in Lemma A.5 is satisfied.

Note that the diffusion matrix of system (3) is

$$
\begin{aligned}
A & =\left(a_{i j}\right)_{n \times n} \\
& =\operatorname{diag}\left(\alpha_{1}^{2} S_{1}^{2}, \beta_{1}^{2} I_{1}^{2}, \sigma_{1}^{2} R_{1}^{2}, \ldots, \alpha_{n}^{2} S_{n}^{2}, \beta_{n}^{2} I_{n}^{2}, \sigma_{n}^{2} R_{n}^{2}\right) .
\end{aligned}
$$

For any bounded domain $D \subset R_{+}^{3 n}$ there is

$$
M=\min \left\{\alpha_{i}^{2} S_{i}^{2}, \beta_{i}^{2} I_{i}^{2}, \sigma_{i}^{2} R_{i}^{2}, i=1,2, \ldots, n, Y \in \bar{D}\right\}>0
$$

such that

$$
\begin{aligned}
\sum_{i, j=1}^{3 n} a_{i j} \xi_{i} \xi_{j}= & \sum_{i=1}^{n} \alpha_{i}^{2} S_{i}^{2} \xi_{3 i-2}^{2}+\sum_{i=1}^{n} \beta_{i}^{2} I_{i}^{2} \xi_{3 i-1}^{2} \\
& +\sum_{i=1}^{n} \sigma_{i}^{2} R_{i}^{2} \xi_{3 i}^{2} \geq M\left\|\xi^{2}\right\|
\end{aligned}
$$

for all $Y \in \bar{D}, \xi \in R^{3 n}$, which implies condition (B.1) is also satisfied. Therefore, by Lemma A.5, the stochastic system (3) has a stationary distribution $\mu(\cdot)$ and it is ergodic.

\section{Appendix}

For the completeness of the paper, in this section, we list some theory used in the upper sections.

(1) Some graph theory (see $[9,24])$ : a directed graph or a digraph $\mathscr{G}=(V, E)$ contains a set $V=\{1,2, \ldots, n\}$ of vertices and a set $E$ of $\operatorname{arcs}(k, j)$ leading from initial vertex $k$ to terminal vertex $j$. A digraph $\mathscr{G}$ is weighted if each $\operatorname{arc}(j, k)$ is assigned a positive weight $a_{k j}$. Given a weighted digraph $\mathscr{G}$ with $n$ vertices, define the weight matrix $A=\left(a_{k j}\right)_{n \times n}$ whose entry $a_{k j}$ equals the weight of $\operatorname{arc}(j, k)$ if it exists, and 0 otherwise. A weighted digraph is denoted as $(\mathscr{G}, A)$. A digraph $\mathscr{G}$ is strongly connected if for any pair of distinct vertices, there exists a directed path from one to the other. A weighted digraph $(\mathscr{G}, A)$ is strongly connected if and only if the weight matrix $A$ is irreducible [25].

Lemma A.1. If $A$ is nonnegative and irreducible, then the spectral radius $\rho(A)$ of $A$ is a simple eigenvalue, and $A$ has a positive eigenvector $\omega=\left(\omega_{1}, \omega_{2}, \ldots, \omega_{n}\right)$ corresponding to $\rho(A)$.
Remark A.2. The result of this lemma is given in P. 27, [25, Theorem 1.4].

The Laplacian matrix of $(\mathscr{G}, A)$ is defined as

$$
L_{A}=\left[\begin{array}{cccc}
\sum_{k \neq 1} a_{1 k} & -a_{12} & \cdots & -a_{1 n} \\
-a_{21} & \sum_{k \neq 2} a_{2 k} & \cdots & -a_{2 n} \\
\vdots & \vdots & \ddots & \vdots \\
-a_{n 1} & -a_{n 2} & \cdots & \sum_{k \neq n} a_{n k}
\end{array}\right] .
$$

Let $c_{k}$ denote the cofactor of the $k$ th diagonal element of $L_{A}$, which has following property.

Lemma A.3 (see [9]). Assume $n \geq 2$.

(1) If $(\mathscr{G}, A)$ is strongly connected, then $c_{k}>0$ for $1 \leq k \leq$ $n$.

(2) The following identity holds:

$$
\sum_{k, j=1}^{n} c_{k} a_{k j} G_{k}\left(x_{k}\right)=\sum_{k, j=1}^{n} c_{k} a_{k j} G_{j}\left(x_{j}\right),
$$

where $G_{k}\left(x_{k}\right), 1 \leq k \leq n$ are arbitrary functions.

Remark A.4. Lemma 2.1 of [8] gives the result (1) of Lemma A.3, and the result (2) is referred to [9, Theorem 2.3].

(2) Some theory about stationary distribution (see [23]): let $X(t)$ be a homogeneous Markov process in $E_{l}$ ( $E_{l}$ denotes Euclidean $l$-space) described by the stochastic equation

$$
d X(t)=b(X) d t+\sum_{r=1}^{k} g_{r}(X) d B_{r}(t) .
$$

The diffusion matrix is

$$
A(x)=\left(a_{i j}(x)\right), \quad a_{i j}(x)=\sum_{r=1}^{k} g_{r}^{i}(x) g_{r}^{j}(x) .
$$

Assumption B. There exists a bounded domain $U \subset E_{l}$ with regular boundary $\Gamma$, having the following properties.

(B.1) In the domain $U$ and some neighbourhood thereof, the smallest eigenvalue of the diffusion matrix $A(x)$ is bounded away from zero.

(B.2) If $x \in E_{l} \backslash U$, the mean time $\tau$ at which a path issuing from $x$ reaches the set $U$ is finite, and $\sup _{x \in K} E_{x} \tau<\infty$ for every compact subset $K \subset E_{l}$.

Lemma A.5 (see [23]). If (B) holds, then the Markov process $X(t)$ has a stationary distribution $\mu(\cdot)$. Let $f(\cdot)$ be a function integrable with respect to the measure $\mu$. Then

$$
P_{x}\left\{\lim _{T \rightarrow \infty} \frac{1}{T} \int_{0}^{T} f(X(t)) d t=\int_{E_{l}} f(x) \mu(d x)\right\}=1
$$

for all $x \in E_{l}$. 
Remark A.6. The proof is given in [23]. Exactly, the existence of stationary distribution with density is referred to Theorem 4.1, P. 119 and Lemma 9.4, P. 138. The weak convergence and the ergodicity are obtained in Theorem 5.1, P. 121 and Theorem 7.1, P. 130.

To validate (B.1), it suffices to prove $F$ is uniformly elliptical in $U$, where $F u=b(x) \cdot u_{x}+(1 / 2) \operatorname{tr}\left(A(x) u_{x x}\right)$; that is, there is a positive number $M$ such that

$$
\sum_{i, j=1}^{k} a_{i j}(x) \xi_{i} \xi_{j} \geq M|\xi|^{2}, \quad x \in U, \quad \xi \in R^{k}
$$

(see Chapter 3, P. 103 of [26] and Rayleigh's principle in [27] Chapter 6, P. 349). To verify (B.2), it is sufficient to show that there exists some neighborhood $U$ and a nonnegative $C^{2}$ function such that and for any $E_{l} \backslash U, L V$ is negative (for details refer to [28], P. 1163).

\section{Acknowledgments}

The work was supported by the Ministry of Education of China (no. 109051), NSFC of China (no. 10971021 and no. 11201008), and Program for Changjiang Scholars and Innovative Research Team in University.

\section{References}

[1] A. Lajmanovich and J. A. Yorke, "A deterministic model for gonorrhea in a nonhomogeneous population," Mathematical Biosciences, vol. 28, no. 3-4, pp. 221-236, 1976.

[2] E. Beretta and V. Capasso, "Global stability results for a multigroup SIR epidemic model," in Mathematical Ecology, T. G. Hallam, L. J. Gross, and S. A. Levin, Eds., pp. 317-342, World Scientific, Singapore, 1986.

[3] Z. Feng, W. Huang, and C. Castillo-Chavez, "Global behavior of a multi-group SIS epidemic model with age structure," Journal of Differential Equations, vol. 218, no. 2, pp. 292-324, 2005.

[4] H. W. Hethcote, "An immunization model for a heterogeneous population," Theoretical Population Biology, vol. 14, no. 3, pp. 338-349, 1978.

[5] W. Z. Huang, K. L. Cooke, and C. Castillo-Chavez, "Stability and bifurcation for a multiple-group model for the dynamics of HIV/AIDS transmission," SIAM Journal on Applied Mathematics, vol. 52, no. 3, pp. 835-854, 1992.

[6] C. Koide and H. Seno, "Sex ratio features of two-group SIR model for asymmetric transmission of heterosexual disease," Mathematical and Computer Modelling, vol. 23, pp. 67-91, 1996.

[7] H. Guo, M. Y. Li, and Z. Shuai, "Global stability of the endemic equilibrium of multigroup SIR epidemic models," Canadian Applied Mathematics Quarterly, vol. 14, no. 3, pp. 259-284, 2006.

[8] H. Guo, M. Y. Li, and Z. Shuai, "A graph-theoretic approach to the method of global Lyapunov functions," Proceedings of the American Mathematical Society, vol. 136, no. 8, pp. 2793-2802, 2008.

[9] M. Y. Li and Z. Shuai, "Global-stability problem for coupled systems of differential equations on networks," Journal of Differential Equations, vol. 248, no. 1, pp. 1-20, 2010.

[10] Z. Yuan and L. Wang, "Global stability of epidemiological models with group mixing and nonlinear incidence rates,"
Nonlinear Analysis. Real World Applications, vol. 11, no. 2, pp. 995-1004, 2010.

[11] M. Y. Li, Z. Shuai, and C. Wang, "Global stability of multigroup epidemic models with distributed delays," Journal of Mathematical Analysis and Applications, vol. 361, no. 1, pp. 3847, 2010.

[12] Z. Yuan and X. Zou, "Global threshold property in an epidemic model for disease with latency spreading in a heterogeneous host population," Nonlinear Analysis. Real World Applications, vol. 11, no. 5, pp. 3479-3490, 2010.

[13] R. Sun, "Global stability of the endemic equilibrium of multigroup SIR models with nonlinear incidence," Computers \& Mathematics with Applications, vol. 60, no. 8, pp. 2286-2291, 2010.

[14] V. Capasso and G. Sero, "A generalization of the KermackMcKendrick deterministic epidemic model," Mathematical Biosciences, vol. 208, pp. 419-429, 1978.

[15] D. Xiao and S. Ruan, "Global analysis of an epidemic model with nonmonotone incidence rate," Mathematical Biosciences, vol. 208, no. 2, pp. 419-429, 2007.

[16] C. Ji, D. Jiang, Q. Yang, and N. Shi, "Dynamics of a multigroup SIR epidemic model with stochastic perturbation," Automatica, vol. 48, no. 1, pp. 121-131, 2012.

[17] E. Beretta, V. Kolmanovskii, and L. Shaikhet, "Stability of epidemic model with time delays influenced by stochastic perturbations," Mathematics and Computers in Simulation, vol. 45, no. 3-4, pp. 269-277, 1998.

[18] E. Tornatore, S. M. Buccellato, and P. Vetro, "Stability of a stochastic SIR system," Physica A, vol. 354, pp. 111-126, 2005.

[19] C. Ji, D. Jiang, and N. Shi, "The behavior of an SIR epidemic model with stochastic perturbation," Stochastic Analysis and Applications, vol. 30, no. 5, pp. 755-773, 2012.

[20] N. Dalal, D. Greenhalgh, and X. Mao, "A stochastic model of AIDS and condom use," Journal of Mathematical Analysis and Applications, vol. 325, no. 1, pp. 36-53, 2007.

[21] H. Liu, Q. Yang, and D. Jiang, "The asymptotic behavior of stochastically perturbed DI SIR epidemic models with saturated incidences," Automatica, vol. 48, no. 5, pp. 820-825, 2012.

[22] X. Mao, Stochastic Differential Equations and Applications, Ellis Horwood, Chichester, UK, 1997.

[23] R. Z. Hasminskii, "Stochastic stability of differential equations," in Mechanics and Analysis, vol. 7 of Stochastic Modelling and Applied Probability, Sijthoff \& Noordhoff, Alphen aan den Rijn, The Netherlands, 1980.

[24] D. B. West, Introduction to Graph Theory, Prentice Hall, Upper Saddle River, NJ, USA, 1996.

[25] A. Berman and R. J. Plemmons, Nonnegative Matrices in the Mathematical Sciences, Academic Press, New York, NY, USA, 1979.

[26] T. C. Gard, Introduction to Stochastic Differential Equations, vol. 114, Marcel Dekker, New York, NY, USA, 1988.

[27] G. Strang, Linear Algebra and Its Applications, Thomson Learning, 1988.

[28] C. Zhu and G. Yin, "Asymptotic properties of hybrid diffusion systems," SIAM Journal on Control and Optimization, vol. 46, no. 4, pp. 1155-1179, 2007 


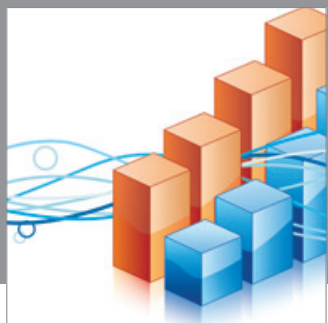

Advances in

Operations Research

mansans

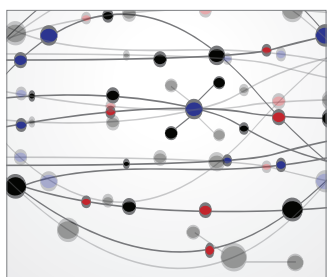

The Scientific World Journal
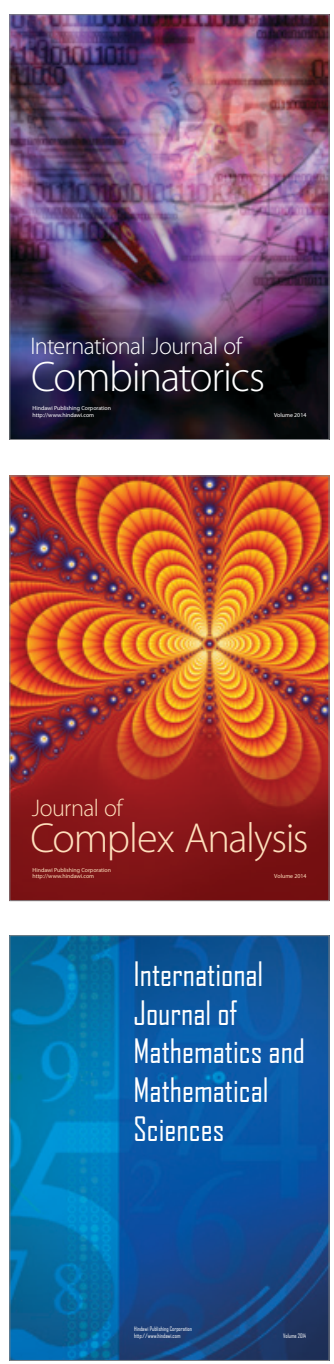
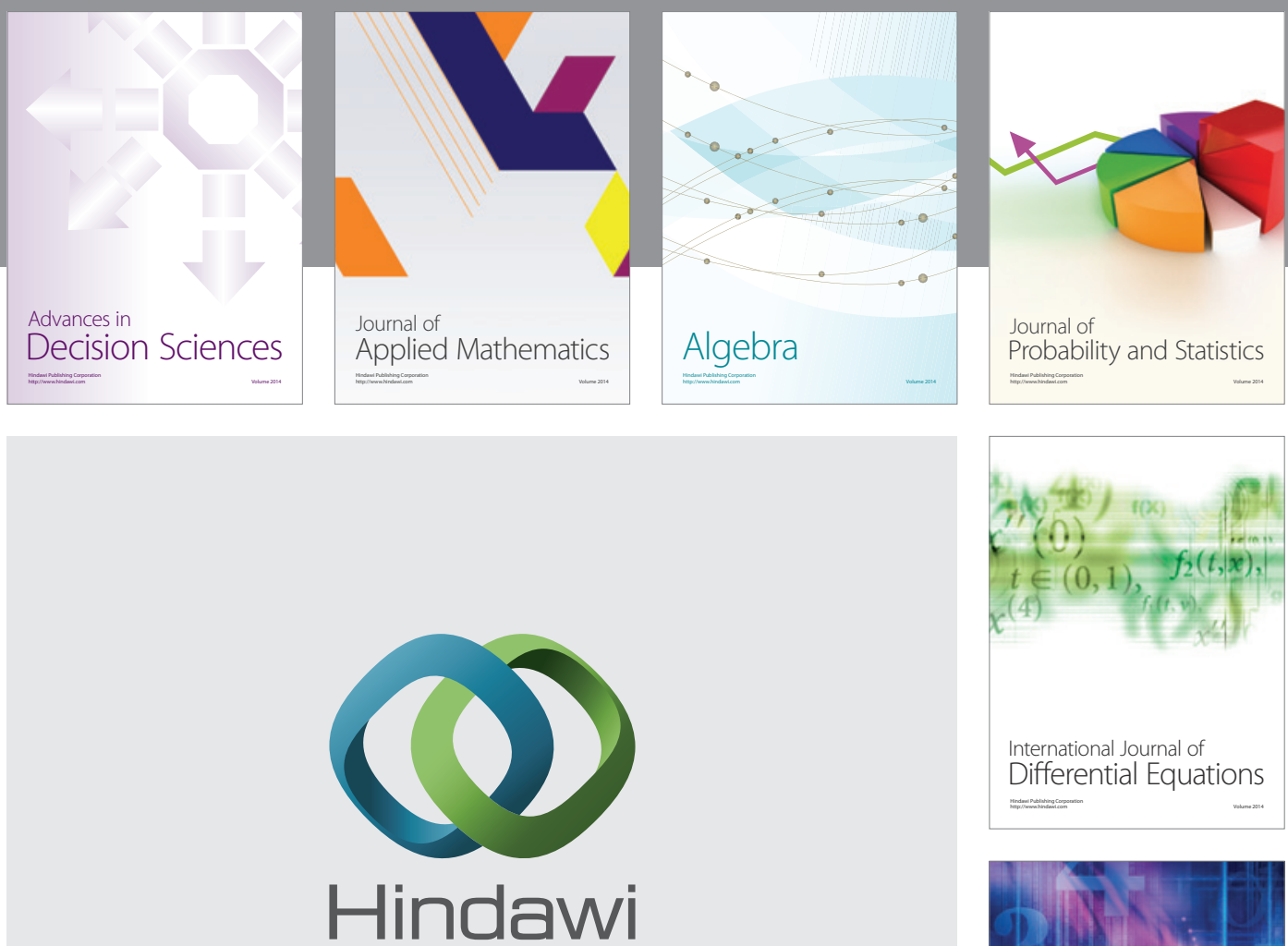

Submit your manuscripts at http://www.hindawi.com
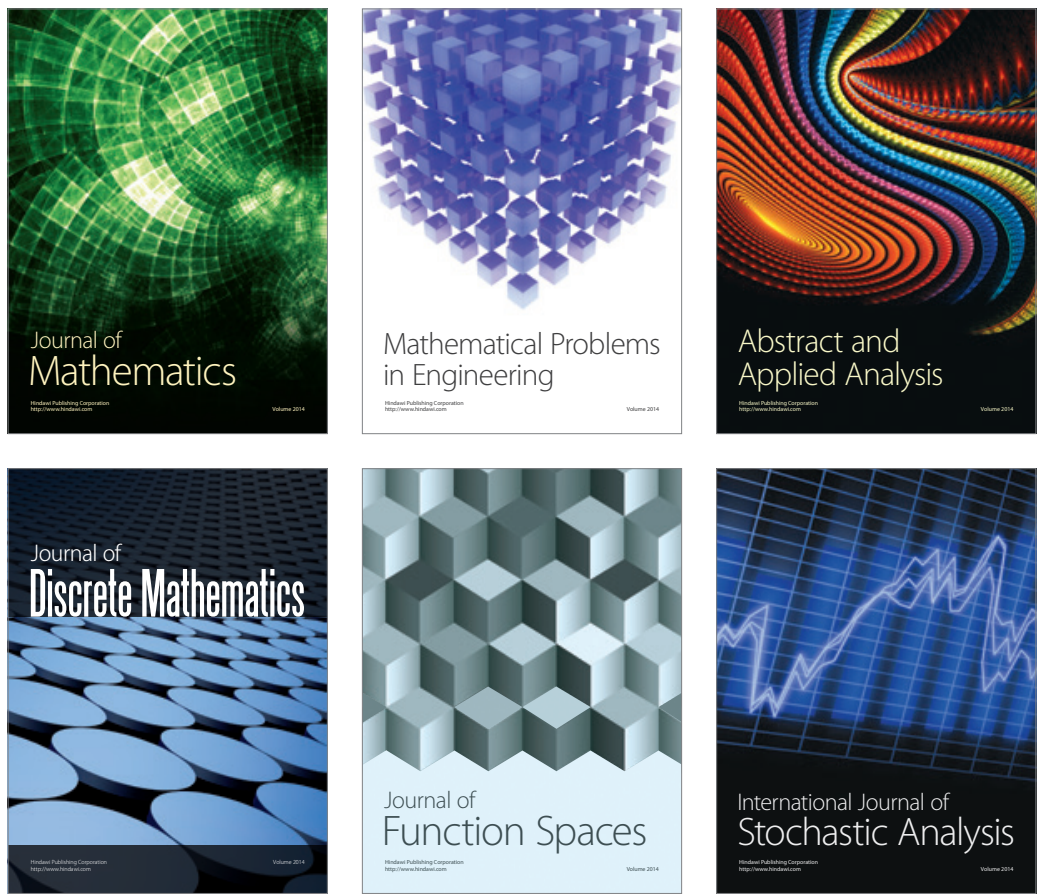

Journal of

Function Spaces

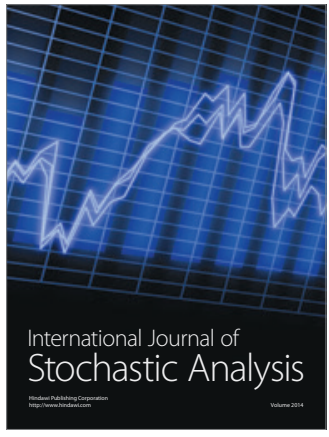

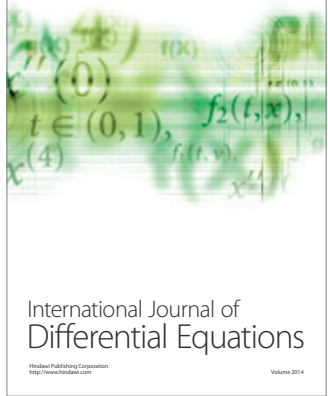
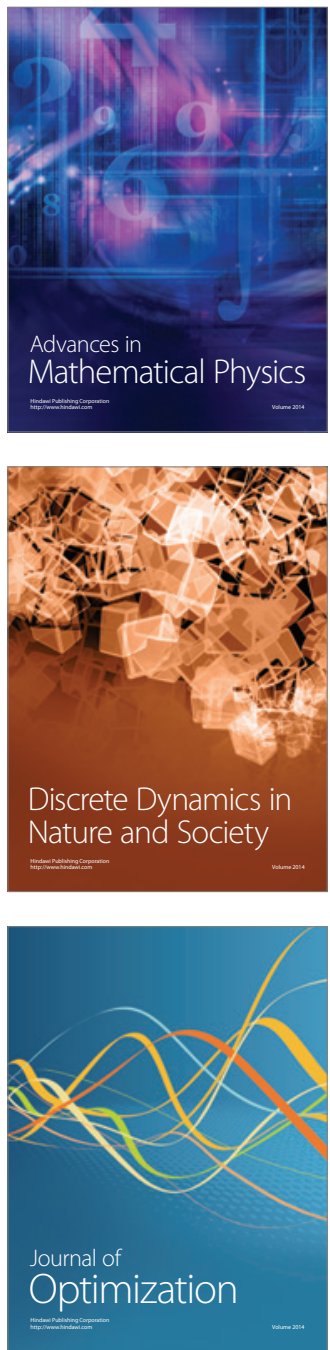